\title{
Mechanical properties and deformation mechanism of Mg-Al-Zn alloy with gradient microstructure in grain size and orientation
}

\author{
Liu Chen ${ }^{1}$, Fuping Yuan, Ping Jiang, Jijia Xie, Xiaolei Wu* \\ State Key Laboratory of Nonlinear Mechanics, Institute of Mechanics, Chinese Academy of Science, Beijing 100190, China
}

\section{A R T I C L E I N F O}

\section{Keywords:}

Gradient structure

Mechanical properties

Tensile ductility

Magnesium alloy

\begin{abstract}
A B S T R A C T
The surface mechanical attrition treatment was taken to fabricate the gradient structure in AZ31 magnesium alloy sheet. Microstructural investigations demonstrate the formation of dual gradients with respect to grain size and orientation, where the microstructural sizes decreased from several microns to about $200 \mathrm{~nm}$ from center area to treated surface, while the $c$-axis gradually inclined from being vertical to treated plane towards parallel with it. According to tensile results, the gradient structured sample has yield strength of $305 \mathrm{MPa}$ in average, which is increased by about 4 times when compared with its coarse-grained counterpart. Meanwhile, contrary to quickly failure after necking in most traditional magnesium alloys, the failure process of gradient structure appears more gently, which makes it has $6.5 \%$ uniform elongation but $11.5 \%$ total elongation. The further comparative tensile tests for separated gradient layers and corresponding cores demonstrate that the gradient structured sample has higher elongation either in uniform or in post-uniform stages. In order to elucidate the relationship between mechanical properties and deformation mechanisms for this dual gradient structure, the repeated stress relaxation tests and pole figure examinations via X-ray diffraction were conducted in constituent gradient layer and corresponding core, as well as gradient structured sample. The results show that the pyramidal dislocations in dual gradient structure are activated through the whole thickness of sample. Together with the contribution of grain-size gradient, more dislocations are activated in dual gradient structure under tensile loading, which results in stronger strain hardening and hence higher tensile ductility.
\end{abstract}

\section{Introduction}

Exploring lightweight materials with high strength and high ductility is clearly attractive but challenging for scientists [1]. Lightweight alloys with high strength have great benefits in energy saving, especially in aeronautical and vehicle fields. However, the strength and ductility are always exclusive for most traditional metals and alloys. For instance, nanostructured metals have strength 10 times higher than their coarse-grained (CG) counterparts due to grain boundary strengthening, but negligible tensile ductility [2,3].

Magnesium alloys are one of the most important lightweight materials and have attracted large amount of interest. One of the roadblocks for their massive application is the low ductility at room temperature. In general, magnesium alloys are of hexagonal closepacked (hcp) crystallographic structure, resulting in quite different deformation mechanisms from high-symmetry materials, e.g., facecentered cubic (fcc) and body-centered cubic (bcc) alloys. There are three main dislocation slip modes for magnesium alloys during plastic deformation, i.e., basal $\langle a\rangle$, prismatic $\langle a\rangle$ and pyramidal $\langle c+a\rangle$. Since the basal $\langle a\rangle$ slip has the lowest critical resolved shear stress (CRSS), it is the easiest one to be activated during deformation. The CRSS of pyramidal $\langle c+a\rangle$ slips are believed significantly higher than that of basal $\langle a\rangle$ slip $[4,5]$. Both basal and prismatic $\langle a\rangle$ slips have no components along $\langle c\rangle$ direction, and only four independent slip systems could be provided by them together, which make it difficult to meet the vonMises criterion for arbitrary deformation [6]. Twinning is another deformation mode for magnesium alloys to accommodate the deformation along $\langle c\rangle$ direction, which has been investigated intensively during past two decades [7-10]. Additionally, stimulating the activation of pyramidal $\langle c+a\rangle$ slip is another effective approach to enhance the dislocation behavior and thus improve the mechanical properties.

The activation of pyramidal slip in magnesium alloys has been observed in many investigations under tensile deformation, e.g., directly changing the crystal structure (i.e., $c / a$ ratio) through alloying with lithium $[11,12]$, increasing the deformation temperature to reduce the CRSS of pyramidal $\langle c+a\rangle$ slip [6], or decreasing the grain size to reduce the yield stress anisotropy between basal and pyramidal slips $[4,13]$. Since grain refining is a more universal strategy which is

\footnotetext{
* Corresponding author.

E-mail address: xlwu@imech.ac.cn (X. Wu).

${ }^{1}$ Present address: Beijing Institute of Aeronautical Materials, Beijing 100095, China.
} 

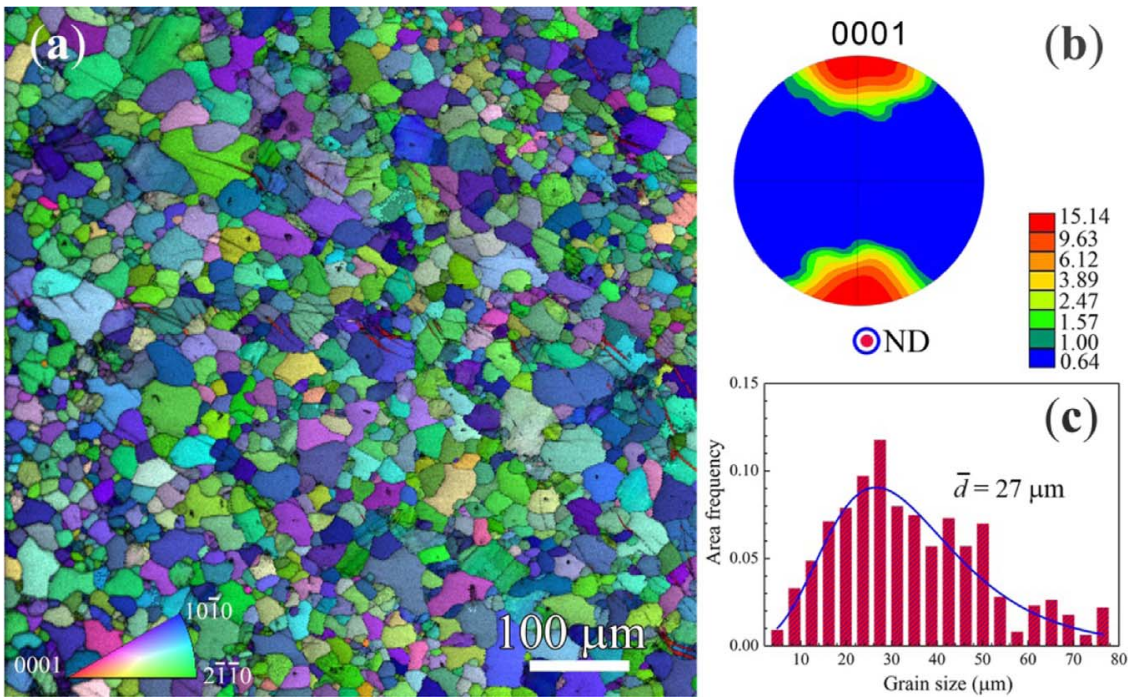

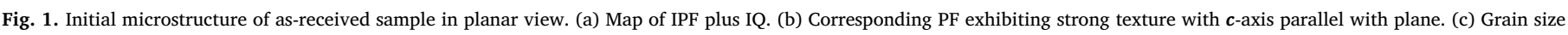
distribution showing an average diameter of $27 \mu \mathrm{m}$.

suitable for different magnesium alloys with a variety of chemical components, it has attracted increasing attention in recent years. As observed in traditional fcc and bcc metals where yield stress increases with decreasing of grain size, the yield stress of magnesium alloys also increases if grain size is reduced, no matter which slip mode dominates their yielding. However, different slip mode displays different sensitivity to grain size change, which apparently leads to different slopes in Hall-Petch equations. Generally speaking, the pyramidal slip has a less slope than basal one, which therefore results in a decreasing anisotropy in yield stress (as characterized by $\tau_{C R S S}^{\text {basal }} / \tau_{C R S S}^{\text {pyramidal }}$ ) with grain refinement. Yu et al. have shown the pyramidal slip in micro magnesium samples with several hundred nanometers in geometry size, demonstrating the enhancement of ductility due to reduction of yield stress anisotropy between basal and pyramidal slips [13]. However, as predicted by trade-off between strength and ductility in traditional metals, simple grain refinement down to nanoscale will significantly lose their ductility since the tiny grains are hardly to store dislocations [14]. Therefore, to retain the favorable contribution due to propensity to trigger pyramidal slip after grain refinement, it is necessary to take strategy to store dislocations inside small grains. Additionally, the activation of pyramidal slip could also be influenced by stress state, where external hydrostatic stress [15] and compatible stress near triple junctions [4] have been observed contributing pyramidal slips.

Recently, it has been evidenced that superior combination between strength and ductility could be achieved through gradient microstructure (GS), where the grain sizes are gradually increased from nanoscale at surface to microscale at center core along thickness direction [16-19]. This microstructure will generate geometrically necessary dislocations (GNDs) during uniaxial stretching due to strain gradient and the change in stress states, which therefore produces an extra strain hardening greater than that given by the mixture law, and results in the enhancement of mechanical properties [18-20]. Meanwhile, the deformation behavior in grains at different depths is quite different due to different yield stresses and strain hardening rates, which leads to strain partitioning and stress transfer between layers at different depths, and thus generates high back stress to improve both strength and ductility [21].

In current investigation, the GS generated through surface mechanical attrition treatment (SMAT) has been employed to improve the mechanical properties of magnesium alloys. This strategy is designed to take advantage of the reduction in yield stress anisotropy due to grain refinement, together with the dislocation accumulation in small grains due to strain gradient and compatible deformation. An AZ31 magne- sium alloy was taken as a model material, which has been extensively investigated with respect to its mechanical property and deformation mechanism. The gradient microstructure and mechanical properties, as well as the change of texture resulting from SMAT and its subsequent influence on plastic deformation have been investigated and analyzed in detail.

\section{Experimental procedures}

The material for this study has a chemical compositions (wt\%) of 3.05Al, $1.04 \mathrm{Zn}, 0.2 \mathrm{Mn}$ and $\mathrm{Mg}$ in balance. The raw materials were melted in electric furnace under protection gas consisting of $\mathrm{CO}_{2}$ and $\mathrm{SF}_{6}$ (99:1 in vol.), then casted into the mold pre-heated to $300{ }^{\circ} \mathrm{C}$. The cast ingot was homogenized at $400{ }^{\circ} \mathrm{C}$ for $10 \mathrm{~h}$, and then hot forged to bar with $90 \mathrm{~mm} \times 50 \mathrm{~mm}$ in cross section and $200 \mathrm{~mm}$ in length. Afterward, the forged bar was annealed at $300{ }^{\circ} \mathrm{C}$ for $30 \mathrm{~min}$ and quenched in water. The plates with thickness of $2 \mathrm{~mm}$ thereafter were sliced out crosswise using electro discharging machining, which are denoted as as-received samples throughout this study.

The SMAT technique was used to produce the gradient microstructure. The surfaces of as-received plates were mechanically polished through silicon carbide abrasive paper to 2000 grit. During SMAT process, 100 no. of stainless steel balls with $3 \mathrm{~mm}$ in diameter were accelerated in chamber by vibrator with frequency of $20 \mathrm{kHz}$, flying toward the sample surface along different directions. Each side of plates had been treated for $15 \mathrm{~min}$ and the final thickness of treated sample is about $1.7 \mathrm{~mm}$.

Tensile tests were conducted using an Instron 5966 test machine at room temperature with strain rate of $5 \times 10^{-4} \mathrm{~s}^{-1}$. Since the as-received plates have strong in-plane (0001) texture as shown in Fig. 1, the dogbone shaped tensile specimen in as-received and SMATed states were cut along two directions with length parallel with and vertical to $c$-axis, which are designated respectively as $\operatorname{CG}\left(0^{\circ}\right)$ and $\mathrm{CG}\left(90^{\circ}\right)$ for as-received sample, or $\mathrm{GS}\left(0^{\circ}\right)$ and $\mathrm{GS}\left(90^{\circ}\right)$ in SMATed state. Tensile samples have gauge sizes of $2.5 \times 18 \mathrm{~mm}^{2}$ in width and length, while three different thicknesses $(1.7,0.2$, and $1.3 \mathrm{~mm})$ were designed for bulk, gradient layer (GL) and corresponding center core. All GLs and cores were obtained from $\mathrm{CG}\left(0^{\circ}\right)$ samples. The engineering strain was measured using extensometer with $5 \mathrm{~mm}$ in gauge length.

The repeated stress relaxation tests were performed using the same Instron machine and extensometer under strain-controlled mode at room temperature with a serial of preset strains. Once attaining any strain with strain rate of $5 \times 10^{-4} \mathrm{~s}^{-1}$, the extensometer strain was 
maintained constant whereas the stress was recorded successively against time. After the first relaxation over an interval of $60 \mathrm{~s}$, the sample was re-stretched by a strain increment of $0.5 \%$ with strain rate of $5 \times 10^{-4} \mathrm{~s}^{-1}$ for next relaxation. Four relaxation cycles were conducted at initial preset strain, and then the sample was strained to next strain at strain rate of $5 \times 10^{-4} \mathrm{~s}^{-1}$. Three times repeat were carried for stress relaxation test, and the calculation methods are referred to references.

The FM100 micro-hardness tester was employed to characterize the distribution of hardness along thickness at cross-section after SMAT. The indenting process was automatically carried under a load of $5 \mathrm{~g}$ with holding time of $15 \mathrm{~s}$, and the tests were repeated for more than ten times at the same depth.

The X-ray diffraction (XRD) was used to investigate the texture at different depth through planar scanning, where the surface layer with proper thickness was removed by mechanical polishing to reach to the prescribed depth. The Rigaku SmartLab diffractometer was employed in this study, using $\mathrm{Cu} K_{\alpha}$ radiation at the voltage of $40 \mathrm{kV}$ and current of $30 \mathrm{~mA}$. The MTEX Toolbox was used to plot the pole figures relating to XRD scanning data [22].

Electron back-scattered diffraction (EBSD) observations were conducted using a field emission JSM 7100F scanning electron microscope (SEM), which equipped with a backscattered electron detector. The samples for EBSD and backscattered image (BSE) examinations were mechanically polished followed by electro-polishing using an electrolyte of $97 \%$ ethylalcohol and $3 \%$ perchloricacid (HClO4) with a voltage of $38 \mathrm{~V}$ at about $-40{ }^{\circ} \mathrm{C}$. The step size $\left(l_{s}\right)$ of EBSD scanning was determined by $l_{s}=d_{a} / N$, where $d_{a}$ is the approximate value of grain diameter, while $N$ is a number ranging from 10 to 20. Accordingly, the step size for as-received sample was set as about $1.5 \mu \mathrm{m}$, while equals about $100 \mathrm{~nm}$ for gradient layers. Additionally, the minimum value of misorientation angle was limited to $2^{\circ}$ during the counting of grain sizes.

Transmission electron microscopy (TEM) observations were conducted through JEM 2010 microscope with an operating voltage of $200 \mathrm{kV}$, and the foil samples for TEM observation were prepared by twin-jet polishing under voltage of $30 \mathrm{~V}$ with the same solution as electro-polishing.

\section{Results}

\subsection{Gradient structure in grain size and texture}

Since magnesium alloys always exhibit strong texture which will then exert strong influence on mechanical properties, the initial texture of as-received samples was characterized by EBSD, and the results are shown in Fig. 1. Fig. 1a displays the map overlapped with inversed pole figure (IPF) and image quality (IQ). Fig. 1b shows the (0001) pole figure $(\mathrm{PF})$, which manifests the strong in-plane texture with $\boldsymbol{c}$-axis parallel with scanning plane. Therefore, two special directions parallel and vertical to $c$-axes will be designated as $0^{\circ}$ and $90^{\circ}$ in suffixes. Fig. $1 \mathrm{c}$ shows the distribution of grain size, and the average value is measured as $27 \mu \mathrm{m}$.

The severely plastic deformation during SMAT will efficiently generate a gradient microstructure with respect to grain size attributed from gradient magnitudes of strain and stress along thickness. Fig. 2 displays the gradient microstructure in grain size and micro-hardness. The size gradient includes the grain size as well as the twin spacing when approaching the center area. As shown in Fig. 2a, the gradient layer with approximate thickness of $400 \mu \mathrm{m}$ has a sharp change of grain size with depth, and furthermore, the deformation twinning have occurred in center area which make the microstructural size much less than its original grain diameter. Fig. $2 \mathrm{~b}$ exhibits the gradient of microhardness, which is in line with the tendency of size gradient. The typical microstructures close to treated surface and center area are shown in Figs. $2 \mathrm{c}$ and $2 \mathrm{~d}$, which are demonstrated by the TEM micrograph and
EBSD IPF map, respectively. Fig. 2c shows a near equiaxed grain of about $300 \mathrm{~nm}$ at depth of $50 \mu \mathrm{m}$, while much larger size of about several microns after partitioning by twin boundaries is exhibited in Fig. 2d.

In respect to texture after SMAT, Fig. 3 displays an important feature different from other fcc and bcc alloys treated by the same technique, where the orientation gradient from center to surface is clearly shown with $c$-axes gradually changing from vertical to parallel directions (schematically illustrated in Fig. 3(f)). The (0001) PFs at different depth were examined by XRD and plotted in Figs. 3a to 3e, which were scanned in planes parallel with surface. The relatively stronger (0001) out-of-plane texture with many $c$-axes nearly parallel with normal directions are shown in Figs. $3 \mathrm{~d}$ and 3e, revealing a $90^{\circ}$ turning of $\boldsymbol{c}$-axis during SAMT. With decreasing of depth towards surface, the $c$-axes become inclined gradually and the corresponding maximum intensities are also decreased, demonstrating the formation of orientation gradient along with grain-size gradient during SAMT. Accordingly, a dual gradient microstructure was generated in magnesium alloys.

\subsection{Mechanical properties}

Fig. 4 shows the mechanical properties according to uniaxial tensile tests. Fig. 4a shows engineering stresses plotted against engineering strains. The as-received samples show different mechanical response when stretch along different directions, which therefore shows lower yield stress (70 MPa) and higher tensile elongation along $c$-axes $\left(\mathrm{GS}^{0^{\circ}}\right)$, but higher yield stress (138 $\mathrm{MPa})$ and lower tensile elongation vertical to $c$-axes $\left(\mathrm{GS}^{90^{\circ}}\right)$. After SAMT, the difference in respect to tensile curve along two directions becomes disappearing, which makes the tensile curves seem nearly overlapped with each other, manifesting the formation of similar microstructure viewed from two directions. In addition, the post-uniform elongation in GS sample is about $5 \%$, which is nearly negligible for as-received sample. Since the voids within twins are prone to formation in coarse grains, it makes the magnesium alloys deformed localized and ending up with failure prior to diffuse necking [23]. However, the GS samples have shown significant elongation after maximum load, revealing higher deformability attributed from gradient microstructure. Actually, the total elongation of GS sample is even higher than that of CG specimen which was loaded vertical to $c$-axis $\left(\mathrm{GS}^{90^{\circ}}\right)$.

The gradient structured sample is always viewed as an integration of different layers with increasing grain sizes along thickness, and the interaction between layers will be generated during deformation due to the requirement of compatibilities across different layers [18,19,24]. In order to reveal the interaction between layers, the GL with thickness of $200 \mu \mathrm{m}$ and corresponding core were prepared and tested, which are shown in Fig. 4b. For comparison, the tensile curves of GS sample and $100-\mu \mathrm{m}$-thick GL are also plotted here. According to the yield stresses of GL and core (320 MPa in GL and $280 \mathrm{MPa}$ in corresponding core), the rule of mixture (ROM) gives an average yield stress of $289 \pm 15 \mathrm{MPa}$, while the GS sample exhibits a real yield stress of $305 \pm 10 \mathrm{MPa}$, revealing a slight higher value than ROM prediction. This result is in alignment with the previous investigations for extra hardening of gradient microstructure $[18,19]$. Furthermore, it should be noted that the uniform elongation of GS samples is amazingly greater than those of both GL and corresponding core, and this phenomenon will be more significant when it comes to the total elongation. The mechanism attributed to this phenomenon will be analyzed later in detail based on the unique dual gradient microstructure in GS samples.

Figs. $4 \mathrm{c}$ and $4 \mathrm{~d}$ display the strain hardening rate corresponding to Fig. 4b, which are plotted against true strain and true stress, respectively. In general, the uniform deformation during tensile tests requires a consistent high strain hardening to balance the geometrical softening due to reduction of cross-sectional area. However, as shown in Fig. 4c, the strain hardening rate of GLs decreases sharply with the increasing of 

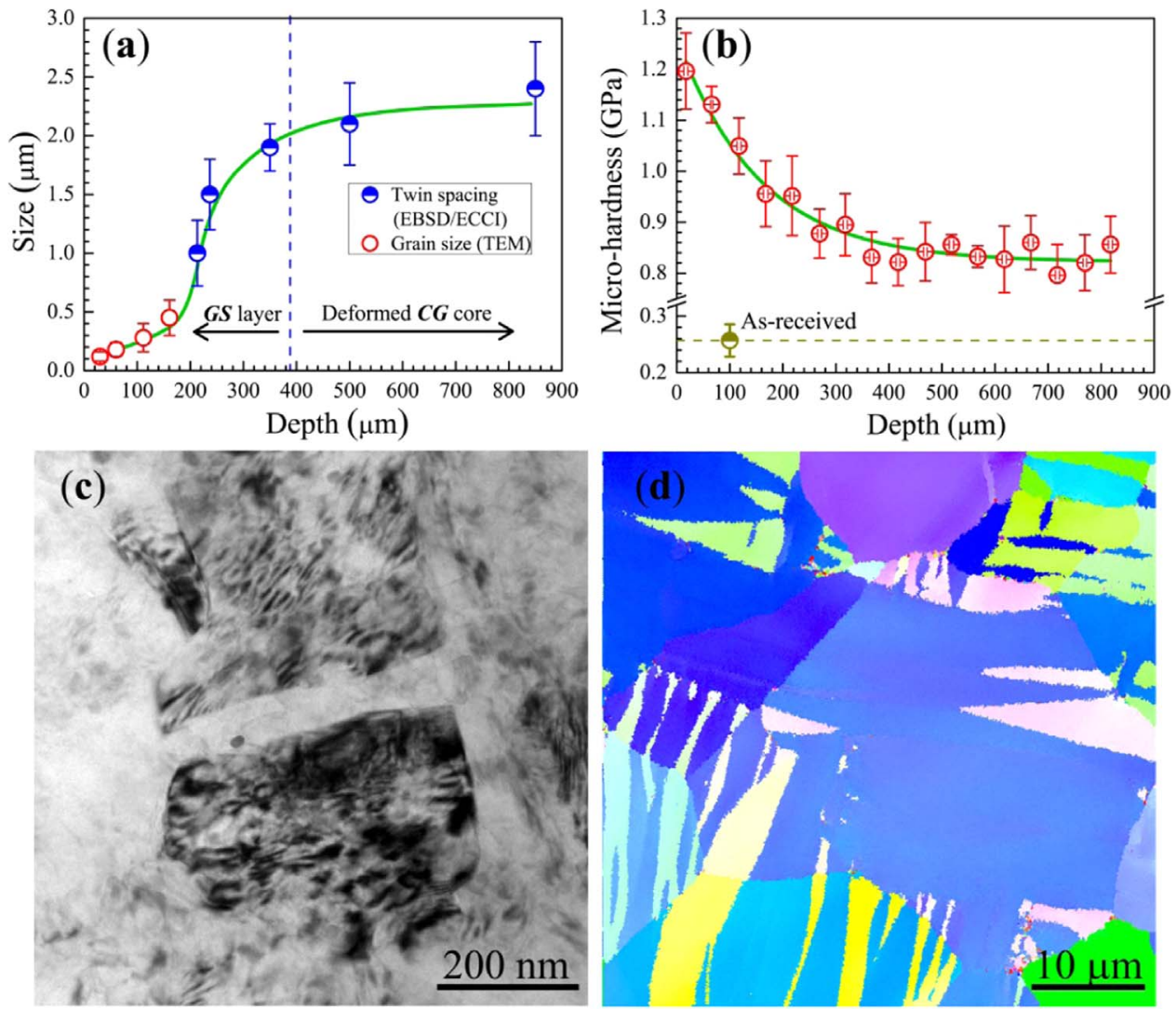

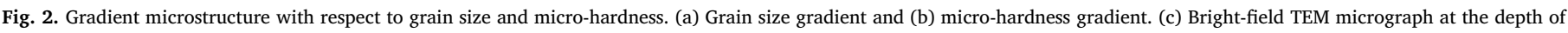

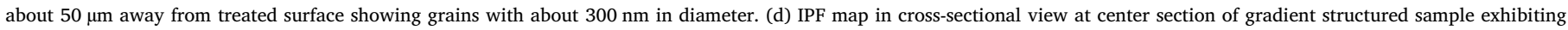
deformation twins and larger grains with diameters of about $30 \mu \mathrm{m}$.

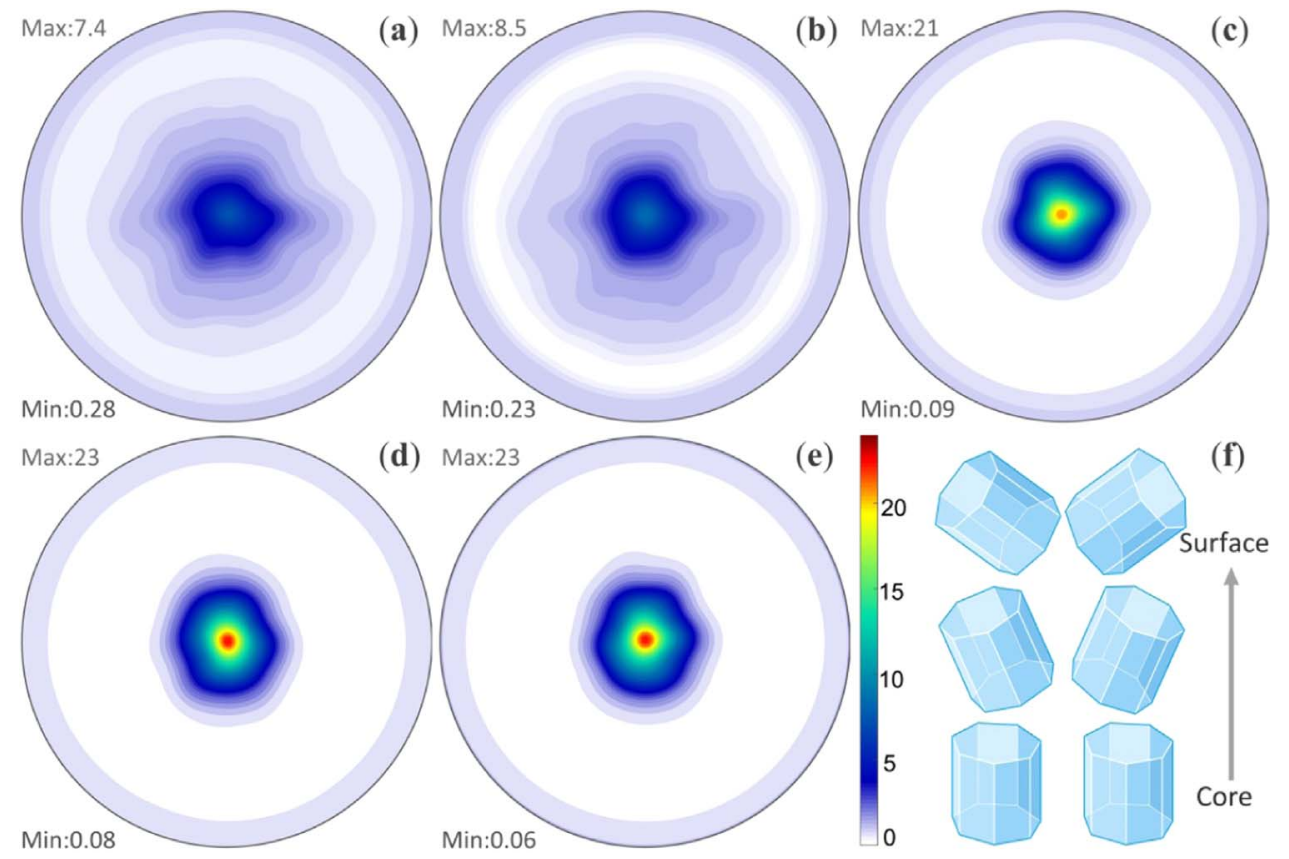

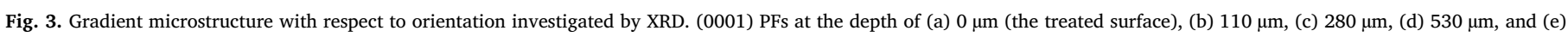

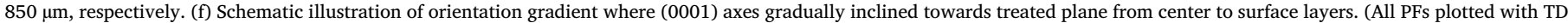
in East and LD in North directions).

strain, which hence triggers the Considere criterion at small strain, leading to low uniform elongation [25]. The GS sample has a moderate strain hardening rate, gently decreasing with the increase of tensile strain, and thus generates the highest uniform elongation. Therefore, not only the level of strain hardening rate itself, but also the decreasing trend with tensile strain, is important to determine the magnitude of uniform elongation. The decreasing of strain hardening rate is always resulted from dynamic recovery, and could be described by the slope of 

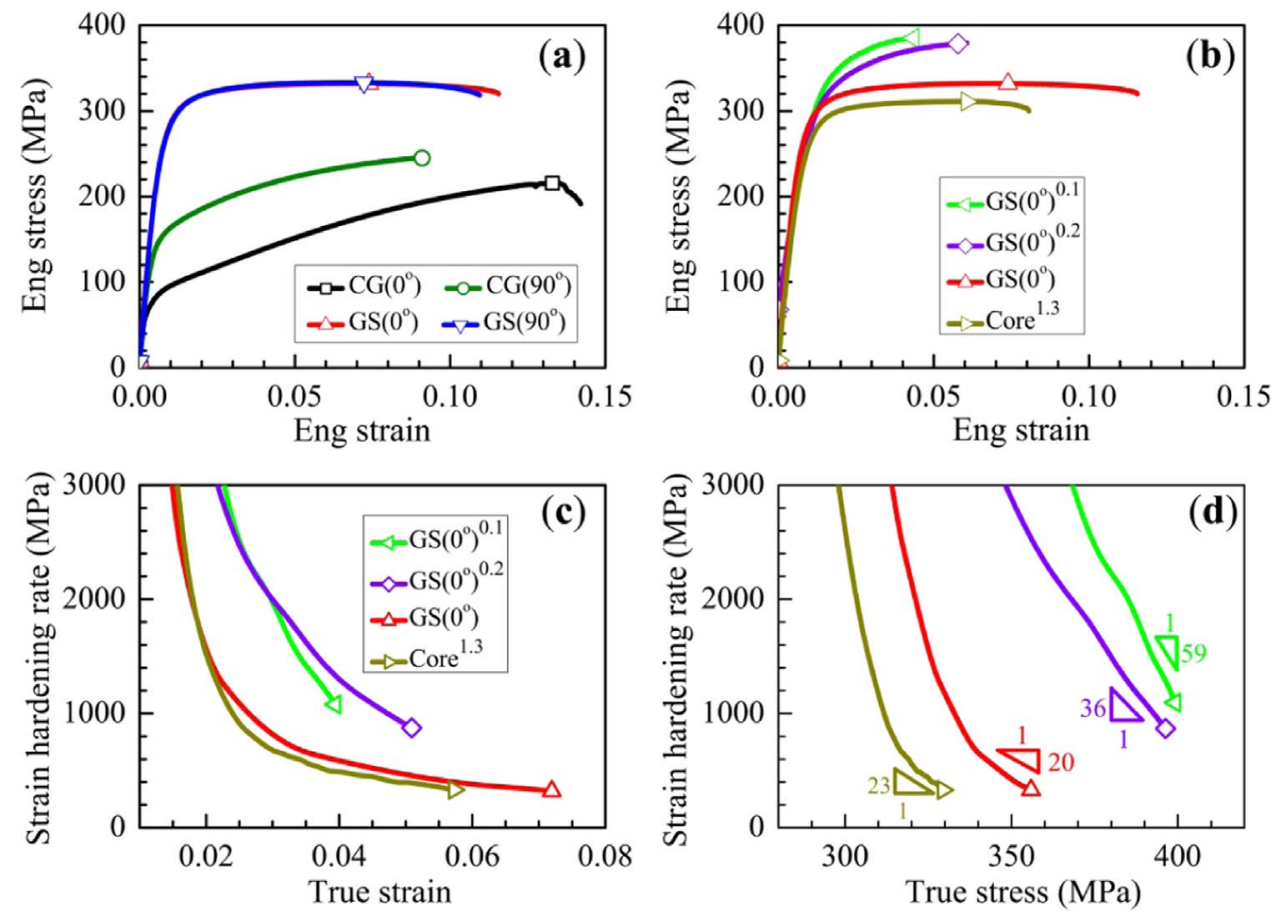

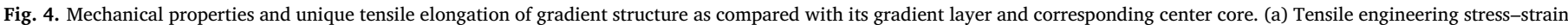

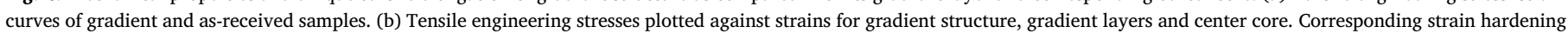
rates plotted against (c) true strain and (d) true stress, respectively.

strain hardening rate curve plotted against true stress [26]. Fig. 4d shows the rates of dynamic recovery corresponding to Fig. 4b, where GS specimen exhibits the lowest rate. The dynamic recovery is generally related to annihilation of dislocations due to cross-slip in CG metals or exhaustion of dislocation due to grain boundary trap in nanostructured metals $[26,27]$, and could be suppressed by the generation of GNDs due to interaction between different phases $[28,29]$. The lowest rate for GS sample manifests that the multiplication of dislocations was not drastically lowered during tensile deformation, thus enable the GS sample to be stretched to higher strain.

\subsection{Repeated stress relaxation tests}

As just mentioned above, the GS samples might have unique ability to enhance the multiplication and interaction of dislocations. Meanwhile, twinning mechanism seems hard to be activated during tensile test in current gradient microstructure due to small grain size and unfavorable orientation, which will be discussed later. Therefore, the evolution of mobile dislocations was examined through repeated stress relaxation test to reveal the strain hardening mechanism of gradient structure.

Fig. 5 displays the measured data from relaxation tests. Fig. 5a shows the engineering stresses plotted against strain for GS, $200-\mu \mathrm{m}$ thick GL and corresponding center core. Their shear stress decays for each first relaxation at different strains are exhibited in Figs. 5b, 5c, and $5 \mathrm{~d}$, respectively. The more the shear stress drops, the larger the plastic deformation occurs in gauge section during relaxation. The shear stress decays are hence related to mobile dislocation behavior, which could be further calculated to obtain the evolution of mobile dislocation density for intuitive viewing.

Fig. 6 exhibits the calculated data of repeated stress relaxation tests according to measured data in Fig. 5. Figs. 6a to $6 \mathrm{c}$ displays the exhaustion curves of mobile dislocation for GS, GL and core samples, where the vertical axes denote the retained fraction of mobile dislocations. With regards to GS sample, the exhaustion curves are rising up in general with increase of tensile strain, demonstrating increasing retained mobile dislocations within gradient microstructure. This is in alignment with previous observations for gradient structured IF steel,
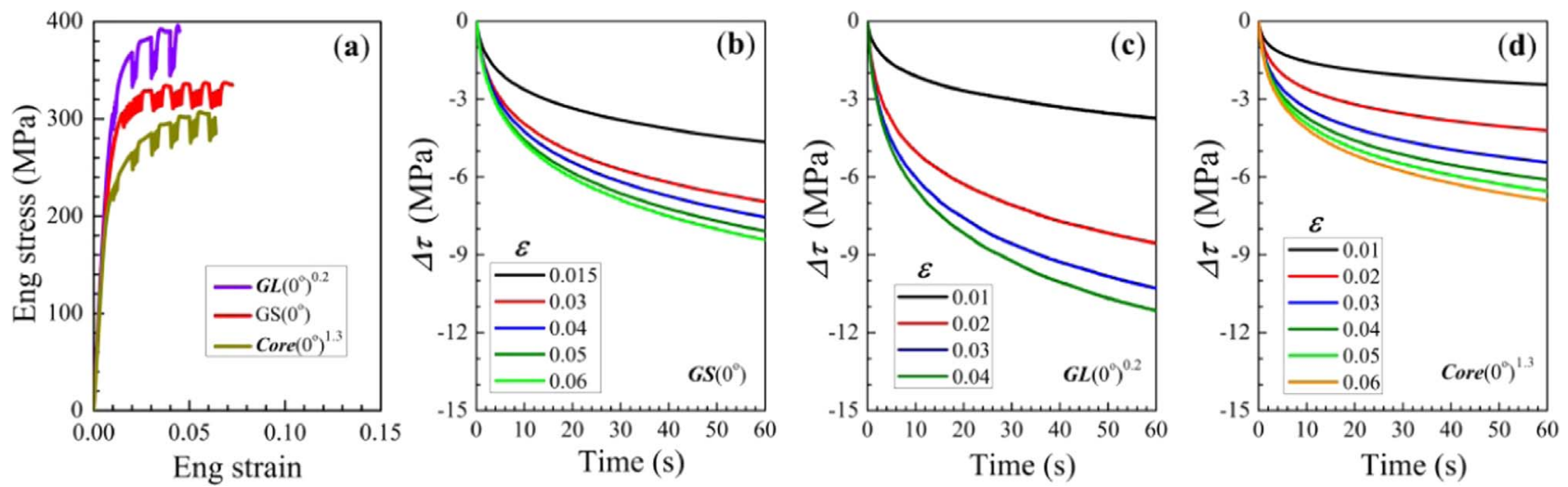

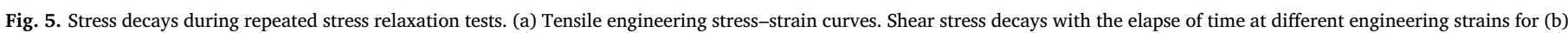
gradient structure, corresponding (c) gradient layer and (d) center core. 

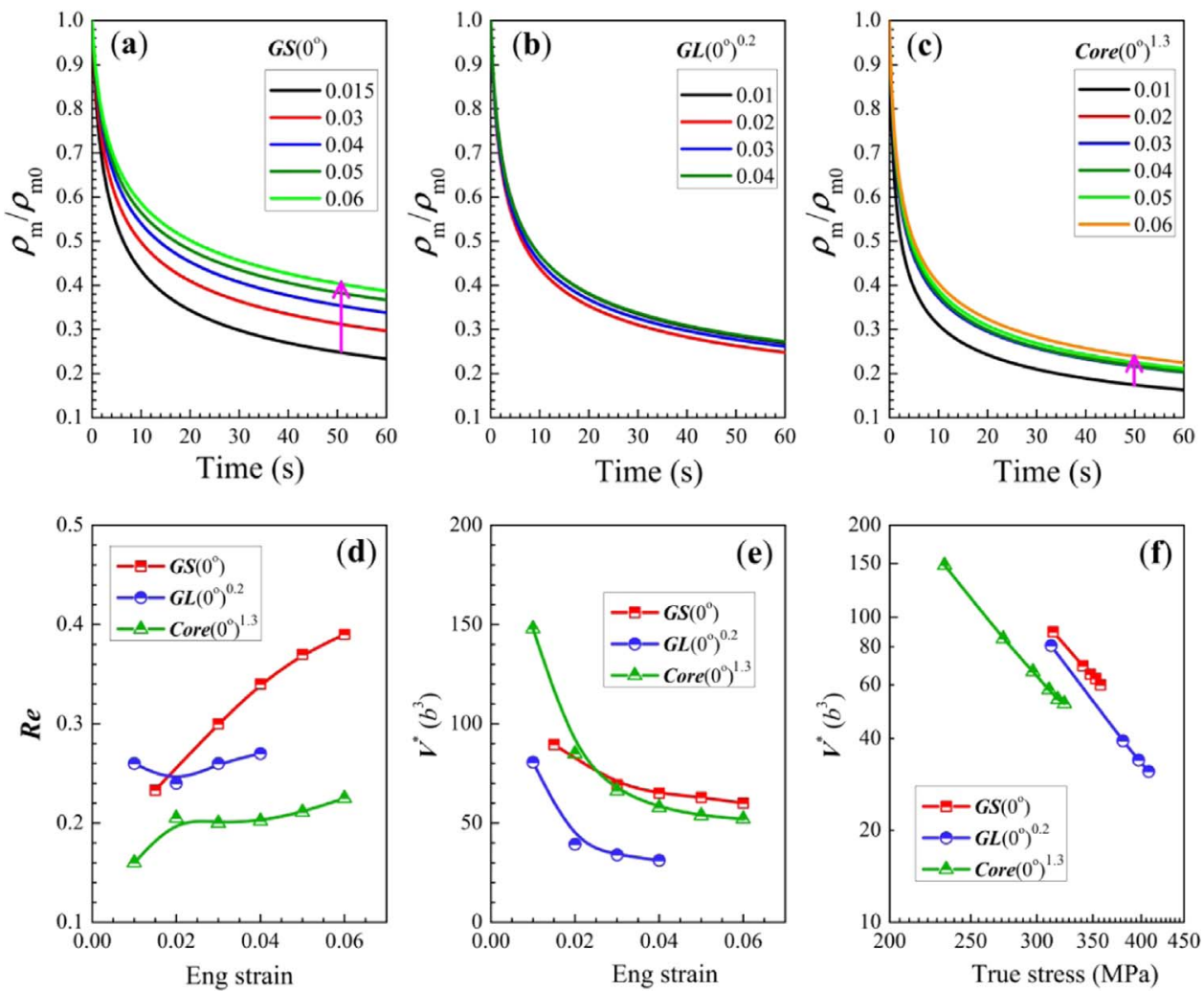

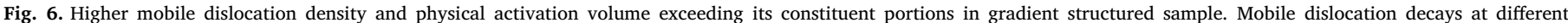

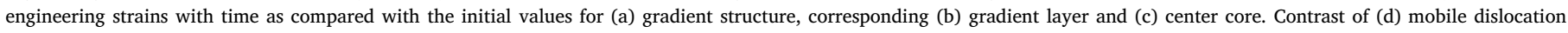

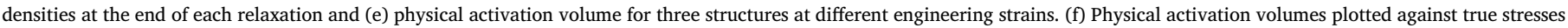
in log coordinate.

which were mainly attributed from the generation of dislocation due to stress state changes and strain gradients [18]. However, the difference between exhaustion curves at different tensile strains for GL specimen is nearly negligible, while a slight increase is observed for center core as shown in Fig. 6c. The retained density of mobile dislocation at the end of relaxation is further plotted in Fig. 6d against tensile strains, which is more intuitive to exhibit the more significant increase of mobile dislocation for gradient structure than those for both GL and corresponding core.

Fig. 6e shows the physical activation volumes of GS, GL and core samples. When tensile strain increases, the physical activation volumes in three structures begin to decrease. In order to understand the physical meaning reflected in this figure, it is necessary to look at what the physical activation volume depends on. According to textbook [30], the equation $V^{*}=b d l$ reveals that the physical activation volume depends on the magnitude of Burgers vector $(b)$, the activation length or size of barrier $(d)$, and the mean free path or distance between barriers $(l)$. Therefore, with increasing of strain, the mean free path decreases due to multiplication of dislocations, and thus leads to reduction of physical activation volume. Accordingly, as evidenced in Fig. 6d, the GS sample which has great ability to produce mobile dislocation should display a lower physical activation volume at least than the ROM predictions. However, as shown in Fig. 6e, the GS sample demonstrates high $V^{*}$ values, which are even higher than the physical activation volumes of center core at strains larger than $2 \%$. Since the activation length is nearly constant for dislocation-dislocation interaction, it is more possible that the magnitude of Burgers vector was changed because different type of dislocation with greater magnitude of Burgers vector was activated in GS sample. It is well-known the pyramidal $\langle c+a\rangle$ dislocations have Burgers vectors significantly longer than basal or prismatic $\langle a\rangle$ dislocations, and hence are energetically less favored $[23,31]$. For instance, in magnesium with $c / a$ ratio of 1.6 , the magnitude of pyramidal dislocation $1 / 3\langle 11 \overline{2} 3\rangle$ is about 1.9 times higher than basal or prismatic dislocation $1 / 3\langle 11 \overline{2} 0\rangle$. Accordingly, the formation of pyramidal dislocations seems the primary reason to make the physical activation volume in GS sample higher than those in GL and core samples.

Fig. $6 \mathrm{f}$ displays the physical activation volumes plotted against true stress on log-log axes. Since the mean free path of dislocation-dislocation interaction has a relationship of [32]:

$l \approx 1 / \sqrt{\rho}$,

where $\rho$ denotes dislocation density, and the stress depends on dislocation density according to:

$\sigma=\alpha M \mu b \sqrt{\rho}$,

where $\alpha, M$ and $\mu$ are constant, Taylor factor and shear modulus, the physical activation volume hence could be expressed as:

$V^{*} \approx \alpha M \mu d b^{2} / \sigma$.

Therefore, for dislocation-dislocation interaction, the curves of $\mathrm{V}^{*}$ versus stress on log-log axes will exhibit linear relationship. Fig. $6 \mathrm{f}$ shows the linear relationship for three different microstructures, manifests that corresponding plastic deformation was dominated by dislocation mechanisms.

\subsection{Microstructure and texture observation}

\subsubsection{Gradient microstructure after SMAT}

Fig. 7 shows the microstructure at center area after SAMT. The IPF map in planar view is displayed in Fig. 7a, while the IPF plus IQ map is exhibited in Fig. 7b, where plenty of deformation bands are visible, manifesting the plastic deformation in even center area. It is different from previous investigations in respect to gradient microstructure for fcc and bcc metals in terms of resultant microstructure, which exhibited 

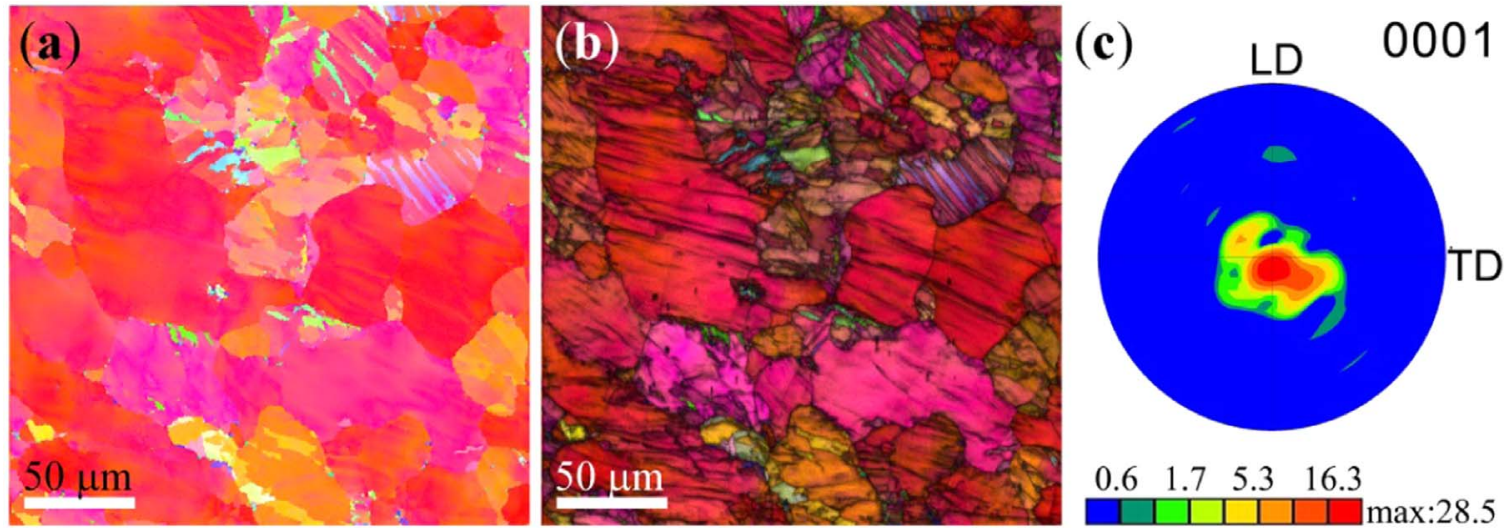

$\begin{array}{llll}0.6 & 1.7 & 5.3 & 16.3\end{array}$
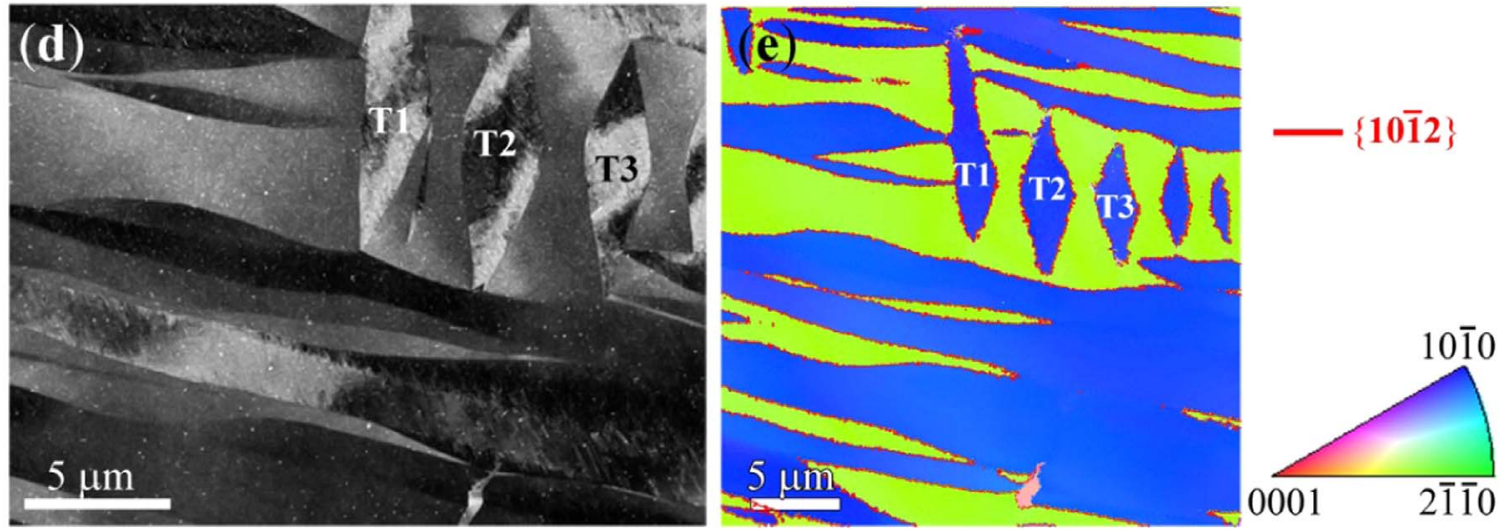

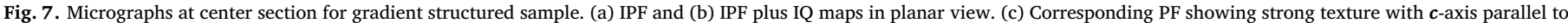

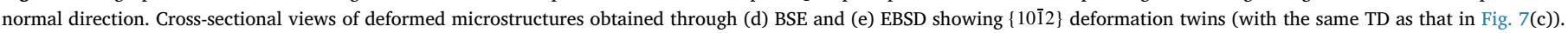

a deformation-free core sandwiched by GLs with thickness of about $200 \mu \mathrm{m}[18,24,33]$. Fig. $7 \mathrm{c}$ is the corresponding PF with transversal direction (TD) in east and loading direction (LD) in north, demonstrating a strong (0001) texture, which is in line with the XRD result shown in Fig. 3e. Figs. 7d and 7e display the BSE micrograph and IPF map in cross-sectional view with higher magnification than Figs. $7 \mathrm{a}$ and $7 \mathrm{~b}$. High density of $\{10 \overline{1} 2\}$ extensive twins is clearly shown, and some of them are marked in Figs. 7d and 7e with symbols of T1, T2 and T3. Given the low stress level to activate extensive twinning (at the same level to basal slip [34]), the center core could be easy to deform
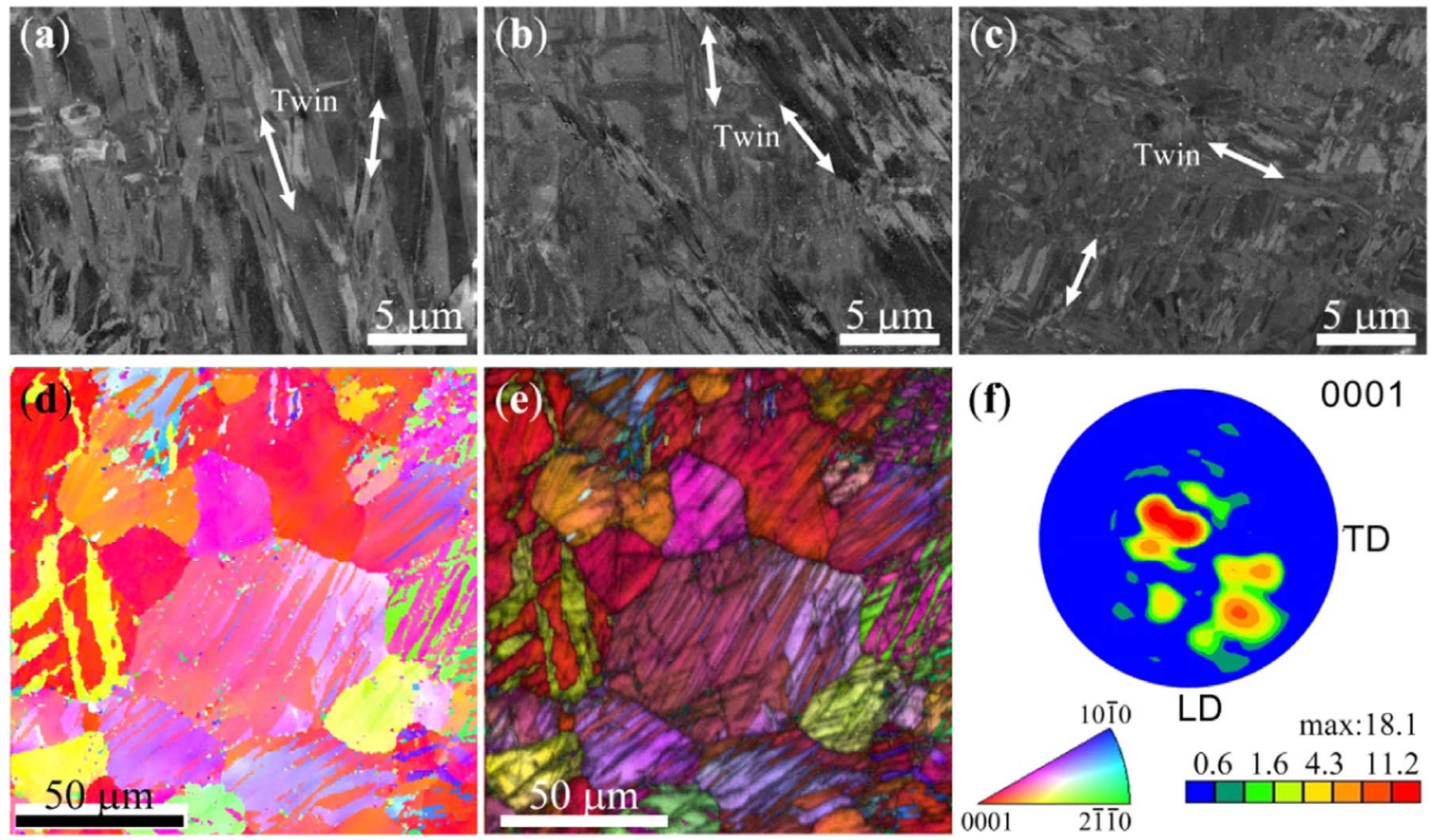

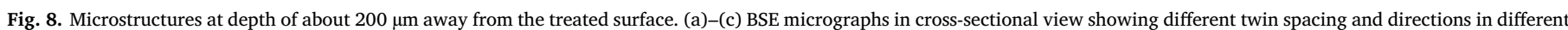
grains. EBSD maps of (d) IPF and (e) IPF plus IQ in planar view, and (f) corresponding (0001) PF. 

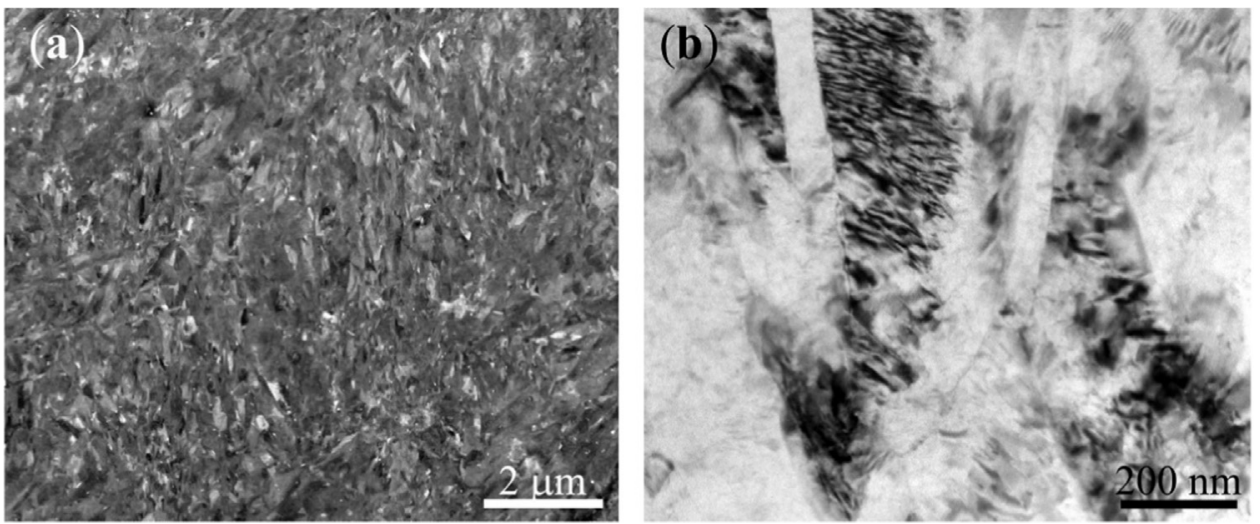

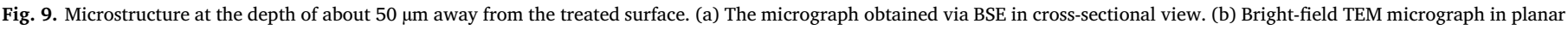
view.

plastically through deformation twinning mechanism during SMAT process.

Fig. 8 displays the microstructure beneath the treated surface of about $200 \mu \mathrm{m}$. Figs. 8a to 8c shows three typical microstructures in cross-sectional view obtained by BSE scanning, where high density of deformation twins on different orientations are exhibited. Since different grains have different crystallographic orientations as compared with the macroscopic coordinate, the twinning propensity hence would be varied from grain to grain, then resulting in different twined microstructure as shown in Figs. 8a to 8c. The deformed microstructure was further examined by EBSD, as shown in Figs. 8d and 8e, which exhibit more severely deformed microstructure than that in center area. Fig. 8 f shows the corresponding PF, which in alignment with the XRD investigations, exhibits a clear divergence of orientations from the center point.

Fig. 9 displays the microstructure beneath the surface of about $50 \mu \mathrm{m}$. Fig. 9a is a BSE micrograph, showing a dramatically deformed microstructure. With TEM observation, as shown in Fig. 9b, the grains of about $200 \mathrm{~nm}$ are formed. Meanwhile, some twins with thickness less than $100 \mathrm{~nm}$ are observable. Within these grains, high density of dislocation was produced due to severely plastic deformation during SAMT.

\subsubsection{Texture change during tensile deformation}

Since the interaction between different layers along thickness is a collective behavior, the texture observation together with previously mentioned stress relaxation was used to analyze the collective change in microstructure and dislocation behavior. According to the relationship between crystal rotation and dislocation slip, the rotation axis can be determined by Taylor axis through the equation of:

$T_{s}=n_{s} \times d_{s}$,

where $T_{s}, n_{s}$ and $d_{s}$ are Taylor axis, slip plane normal and slip direction, respectively. The Taylor axes have been calculated in many investigations and used to analyze which slip mode was activated primarily [19-21]. Based on this theory, the prismatic $\langle a\rangle$ slip produces a rotation axis of $\langle 0001\rangle$, while basal $\langle a\rangle$ and pyramidal $\langle c+a\rangle$ have the same $\langle 10 \overline{1} 0\rangle$ as their Taylor axes. Fig. 10 shows the pole figures of gradient layer and center core before and after tensile deformation. A contraction of contour along LD is observed in (0001) pole figure for gradient layer, revealing a rotation history around TD. Given the inclined orientation in this layer as shown in Fig. 10a, only Taylor axis of $\langle 10 \overline{1} 0\rangle$ could be parallel with TD, which therefore manifests the activation of basal $\langle a\rangle$ or pyramidal $\langle c+a\rangle$ slips in gradient layer. In comparison, as shown in Figs. 10c and 10d, the (1010) pole figure of core sample shows a rotation of contour around ND, which has been evidenced nearly parallel with $\langle 0001\rangle$ direction for core sample (Fig. 3). Hence, this rotation around ND demonstrates the activation of pris-
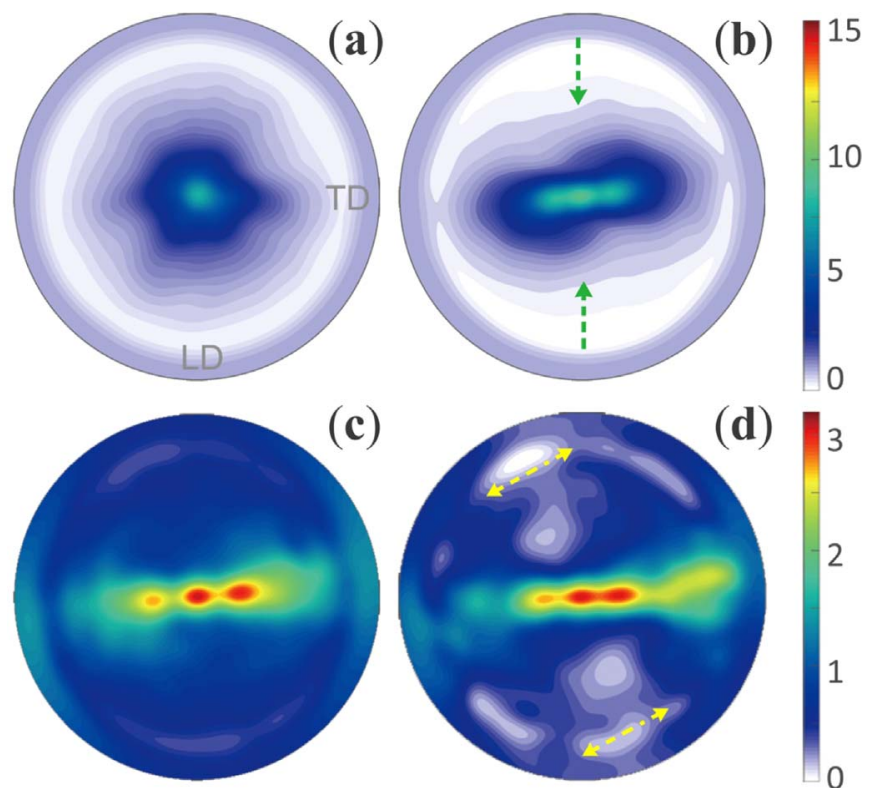

3

Fig. 10. Deformation mechanisms of the standalone gradient layer and center core. (a) and (b) displaying the (0001) PFs on treated surface for standalone gradient layer before after tensile deformation. (c) and (d) showing (1010) PFs on its surface for corresponding core prior to and after tensile deformation. (All PFs plotted with TD in East and LD in North directions).

matic $\langle a\rangle$ slip in the core sample during tensile deformation.

Fig. 11 shows the PFs at different depths before and after tensile deformation. The left column displays the PFs before stretching, while the corresponding figures after deformation are exhibited in right column for comparison. A vertical contraction along LD is clearly seen in each figure on right column, demonstrating the occurrence of basal $\langle a\rangle$ or pyramidal $\langle c+a\rangle$ slips through the entirety of thickness during tensile deformation. Since the texture near center area is initially favorable to prismatic $\langle a\rangle$ slip, the occurrence of strong basal or pyramidal slip features manifests new slip mode was activated in dual gradient microstructure. According to the analysis before, considering the relaxation results and the favorable orientation, the mode should be the pyramidal slip. In addition, the maximum value of intensity at all depths has been increased after deformation, which is alignment with the vertical contraction due to a rotation towards the center point.

\subsubsection{Non-basal dislocation observation}

Fig. 12 displays the TEM micrographs for investigation of $\langle c+a\rangle$ dislocations in gradient microstructure. Fig. 12a shows a bright-field micrograph in planer view at the depth of about $30 \mu \mathrm{m}$ after $5 \%$ elongation. The areas enclosed by symbols of solid circle and asterisk in 


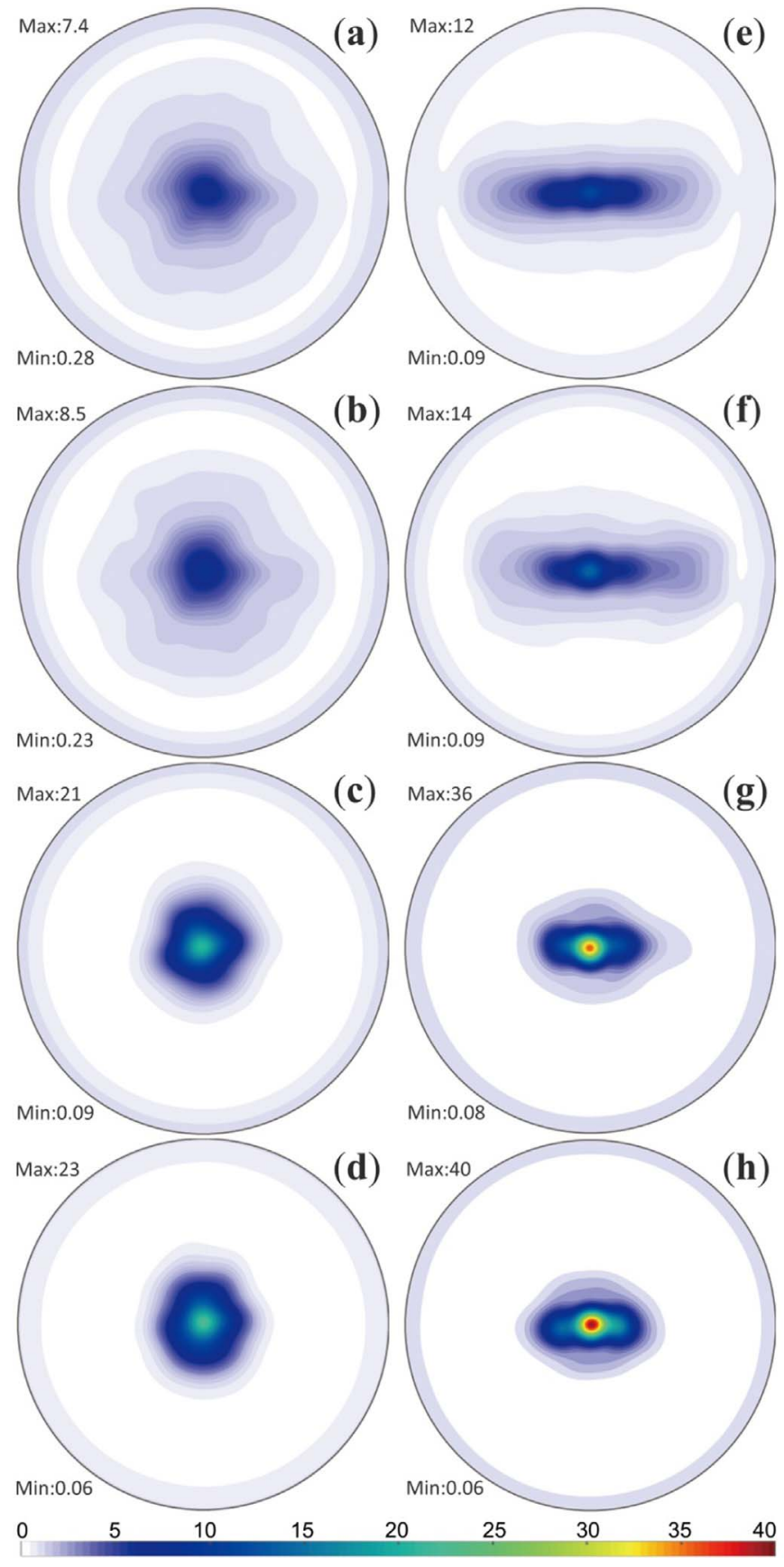

Fig. 11. Deformation mechanisms of GS sample. (a) -(d) (0001) PFs at depth of $0 \mu \mathrm{m}$, $110 \mu \mathrm{m}, 280 \mu \mathrm{m}, 530 \mu \mathrm{m}$ and $850 \mu \mathrm{m}$. Corresponding PFs after 5\% elongation are shown in (e) - (h). (All PFs plotted with TD in East and LD in North directions).

Fig. 12a are correspondingly shown in Figs. 12b and 12c, which were taken under weak-beam dark field conditions with diffraction vector of $\boldsymbol{g}=0002$ and $\boldsymbol{g}=01 \overline{1} 0$, respectively.

When the diffraction vector is $\boldsymbol{g}=0002$, both basal and prismatic $\langle a\rangle$ dislocations are invisible or just exhibit extremely weak contrast, since the condition of $\boldsymbol{g} \cdot \boldsymbol{b}=0$ will right set in for $\langle a\rangle$-type (basal or prismatic $\langle a\rangle)$ dislocations having Burgers vector of $1 / 3\langle 11 \overline{2} 0\rangle$. Accordingly, the dislocations shown in Fig. $12 \mathrm{~b}$ are pyramidal $\langle c+a\rangle$ dislocations. If the diffraction vector is $g=01 \overline{1} 0$ as shown in Fig. 12c, either the $\langle a\rangle$-type or the pyramidal $\langle c+a\rangle$ dislocation could be visible if the Burgers vector not vertical to $\langle 01 \overline{1} 0\rangle$, such as basal $1 / 3\langle 11 \overline{2} 0\rangle$, prismatic $1 / 3\langle 1 \overline{2} 10\rangle$ and pyramidal $1 / 3\langle 1 \overline{2} 13\rangle$. Therefore, TEM observation demonstrates the activation of pyramidal dislocations in gradient structure along with the activation of basal dislocations.

\section{Discussion}

\subsection{Formation of dual gradient microstructure}

With respect to microstructure, the most important feature in GS magnesium alloy is the simultaneous formation of size and orientation gradients, which is called as dual gradient microstructure throughout this manuscript. Since the mechanical properties of magnesium alloys strongly depend on texture, the orientation gradient would lead to the transition of deformation mechanism from layer to layer. Together with the contribution of size gradient, the dual gradient microstructure might enable the magnesium alloy to be stretched with optimized mechanical properties.

The formation of size gradient during SAMT has been well investigated for large amount of fcc, bcc and hcp metals, which is mainly attributed from twinning and dislocation mechanisms. According to microstructure observations, the twinning mechanism have been activated through whole thickness during SMAT processing (Fig. 7 to Fig. 9), which plays an important role in refining the coarsegrained microstructure. Along with the formation and evolution of dislocation boundaries (Fig. 9), the size gradient microstructure was finally produced in magnesium alloy.

Meanwhile, as evidenced in Fig. 3, the orientation gradient was formed during SAMT processing. In comparison with the initial texture (Fig. 1b), the $c$-axes in center area have a change in orientation of about $90^{\circ}$ from in-plane direction towards normal direction, as demonstrated by PFs in Fig. 3d and Fig. 7c. Given the prevalent $\{10 \overline{1} 2\}$ twinning in center area (Figs. 7d to 7e), it is naturally inferred that the change of orientation was resulted directly from the extensive twinning. That is because the extensive twinning will lead to a rotation of $c$-axes around $\langle 11 \overline{2} 0\rangle$ for $86.3^{\circ}$ [18], which is in alignment with the abrupt change of orientation after SMAT processing. Meanwhile, it should be noted that the initial texture in as-received sample is also favorable to extensive twinning, since the peening during SAMT process could generate a compressive stress vertical to $\langle 0001\rangle$ direction, which is evidenced to be the easy mode to activate extensive twinning in magnesium alloys. Therefore, the extensive twinning prevalent in this study is the primary reason resulting in the strong (0001) texture with $\boldsymbol{c}$-axes normal to plane. Afterwards, with the increasing of strain and stress amplitude near treated surface, the dislocation multiplication and interaction as well as subsequent dislocation boundaries would weaken the (0001) texture gradually, and thus results in the orientation gradient along thickness.

\subsection{Influence of grain size on deformation mechanism}

Towards the understanding of deformation mechanisms in this dual gradient microstructure, it is necessary to figure out the influence of grain size on the plastic deformation mechanisms. With regards to dislocation mechanism, the small grains are supposed to be less anisotropic in plastic deformation since the CRSS of prismatic or pyramidal slip is close to the same level of that for basal slip $[13,35]$. It is still interesting and important to ask whether there is a critical size under which the non-basal slips are prevalent. For nanosized particle or tensile sample with single crystal structure, it was found that such critical size for magnesium is about $100 \mathrm{~nm}$ [13,35]. However, in polycrystalline AZ31 magnesium alloy, Koike et al. have observed nonbasal slips activated in grains with average diameter of $6 \mu \mathrm{m}$ [4]. As supposed by the authors, compatibility across grain boundaries would raise the local stress and hence reduce the yield anisotropy substantially, leading to the activation of pyramidal slips to accommodate the possible incompatibilities. Therefore, the critical size for triggering nonbasal slip depends on the chemical composition, local stress state and so on. Anyway, the refined microstructure of magnesium alloys is definitely beneficial to non-basal slips, which therefore improves the ability of uniform deformation. 

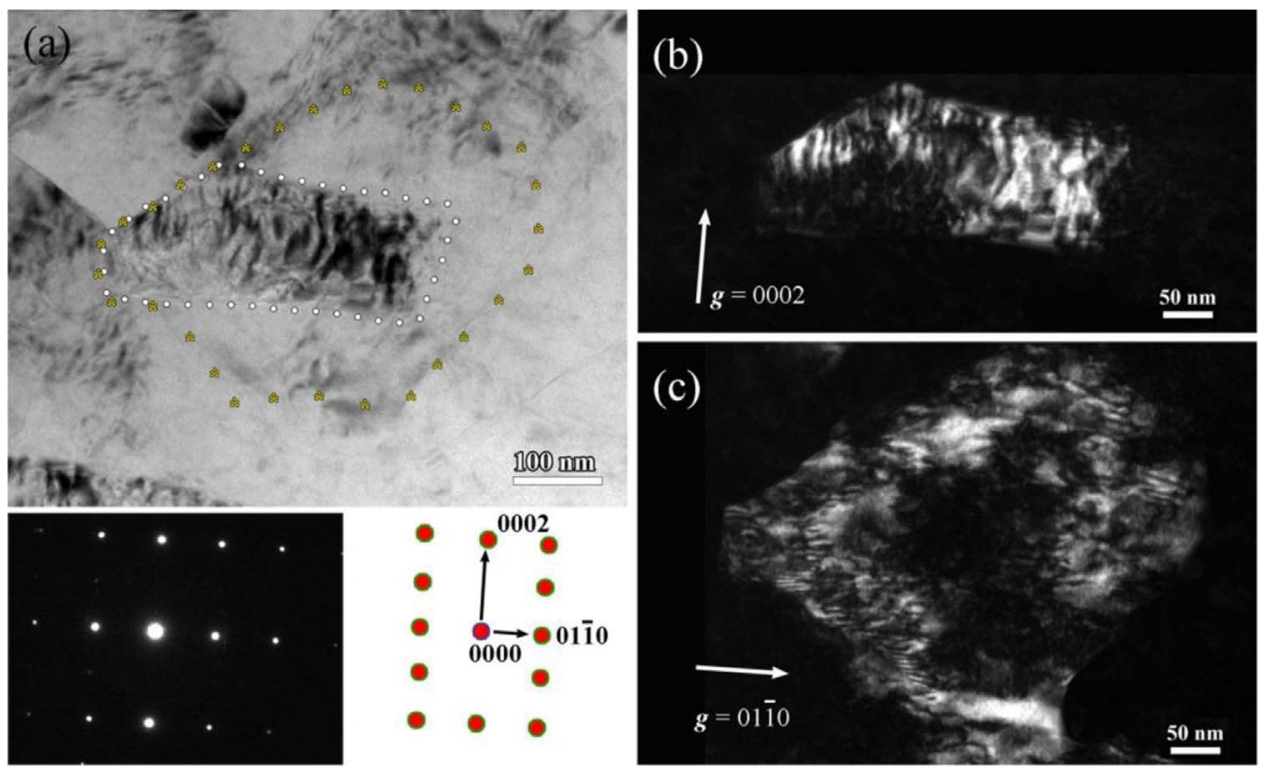

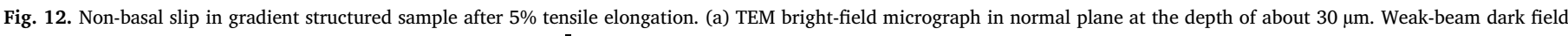
micrographs using diffraction vector of (b) $g=0002$ and (c) $g=01 \overline{1} 0$.

In respect to twinning mechanism, it also depends on grain size for magnesium alloys. Broadly speaking, the extensive twinning is always activated in larger grains $[14,36]$. No twinning was shown during micropillar compression for single crystal magnesium with diameters of several microns [37]. With the further decreasing of size, as indicated by pillar diameter, deformation twinning would reappear like what have been well observed in fcc metals. However, the critical size under which deformation twinning was observed is always less than $200 \mathrm{~nm}$, and the critical stress for twinning nucleation is higher than $1 \mathrm{GPa}$ $[16,38]$. Therefore, given that the most fractions of grains in dual gradient microstructure of this study is greater than that value (Fig. 2a), the deformation twinning during tensile deformation would be negligible, and thus the deformation mechanism is mostly related to dislocation slips.

Accordingly, for the dual gradient microstructure in present investigation, the plastic deformation is dominated by dislocation slip rather than deformation twinning. The grain refinement along thickness could play an important role in decreasing the yield anisotropy during tensile deformation, and hence enhance the propensity for pyramidal slips to accommodate deformation along $\boldsymbol{c}$-axes.

\subsection{Influence of orientation and its gradient on deformation mechanism}

Owing to the low-symmetric crystallographic structure for hcp metals, their deformation mechanisms activated during tensile deformation and the subsequent mechanical properties are closely dependent on the loading direction. In general, when stretched along or compressed vertical to $\boldsymbol{c}$-axes, extensive twinning is the easiest mode to be activated. When deformed with loading inclined to $c$-axes, the basal $\langle a\rangle$ slip will be activated firstly. Additionally, the prismatic $\langle a\rangle$ slip will be triggered if the tensile loading has a direction vertical to $c$-axes [18].

In current study, the treated surface and center area differ clearly in texture, where $\boldsymbol{c}$-axes tend to be tilted to normal direction near treated surface, but show a trend parallel with normal direction at center area. Therefore, in terms of plastic deformation mechanism, the gradient layer with more tilted $\boldsymbol{c}$-axes would be more favorable to basal $\langle a\rangle$ slip, while the prismatic $\langle a\rangle$ slip will dominate the deformation mechanism in center core due to vertical $c$-axes to loading direction. The difference in dominant slip modes between gradient layer and center core has been evidenced through the investigation of pole figures (Fig. 10). The vertical contraction of contour towards center point in (0001) pole figure of gradient layer is attributed from crystal rotations along TD (Fig. 10b), which reveals the basal $\langle a\rangle$ or pyramidal $\langle c+a\rangle$ slip with Taylor axis of $\langle 10 \overline{1} 0\rangle$. In contrast, the rotation around ND in (1010) pole figure of core sample was observed (Fig. 10d), demonstrating the prismatic $\langle a\rangle$ slip with Taylor axis of $\langle 0001\rangle$. Therefore, the dominant slip modes are exactly changed along thickness.

Meanwhile, the different dominant mechanisms between gradient layer and core due to orientation are also evidenced by the Hall-Petch relationship. According to the available data [39-44], as shown in Fig. 13, when grain size is greater than $1 \mu \mathrm{m}$, the lower slope of basal slip mechanism manifests that this deformation mechanism has lower grain size sensitivity with respect to yield strength [41]. The CG samples and the core of gradient structure have different deformation mechanisms due to different textures. While the grain size is less than $1 \mu \mathrm{m}$, the linear relationship begins to deviate and shows a lower slope, which has been ascribed to the transition of dominant deformation mechanism from prismatic to basal slip [44]. Therefore, it manifests that the plastic deformation mechanisms of different layers in gradient microstructure are changed with depth due to different orientations.

For gradient microstructure, which could be viewed as a pile of layers with gradually changed size and orientation, the difference in

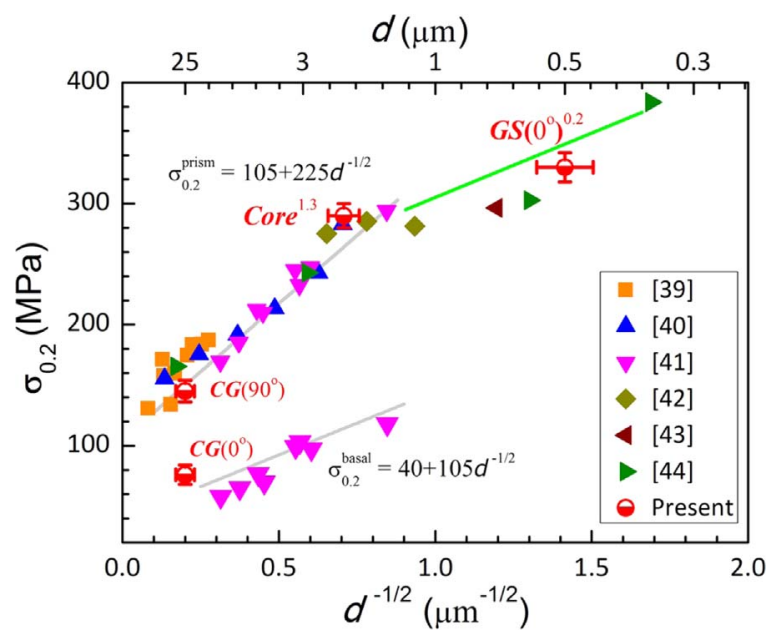

Fig. 13. The yield stresses of different magnesium alloys plotted against grain size showing Hall-Petch relationship and corresponding deformation mechanisms. 
deformation modes across layers will generate internal stress in material to remain continuous during plastic deformation. Similar to the grain boundary, as supposed by Koike et al., the compatible stress across layers will significantly improve the activation of non-basal slips [4]. Therefore, for current gradient microstructure (Fig. 3), the difference in orientation along thickness will definitely generate internal stresses to accommodate such anomalously dominant slip modes across layers, and hence, in agreement with the investigations of stress relaxation (Fig. 6), the pyramidal slip is activated.

\subsection{Coupling between size gradient and orientation gradient}

As demonstrated that size and orientation gradients exist together in magnesium alloy, it is interesting to ask how the two gradients affect to or couple with each other. The size-gradient microstructure has been evidenced that its strain hardening can be enhanced through the effects of strain gradient and back stress $[18,45]$. Both the strain gradient and back stress effect are relatively insensitive to materials in respect to the triggering principle, because the strain gradient and subsequent stressstate change are caused by the propensity to instability for layers near to surface, while the back stress is resulted from the heterogeneous nature in strength along thickness. Therefore, such beneficial effects are still work in magnesium alloy with size-gradient structure.

Besides the above effects, since the microstructural size of magnesium alloy was greatly refined along the entirety of thickness, the yield stress anisotropy would be significantly reduced. Accordingly, the sizegradient microstructure has another effect that is to improve the probability in activating pyramidal slip. As compared with the basal $\langle a\rangle$ and prismatic $\langle a\rangle$ slips, the pyramidal slip is the only one that could provide accommodation for deformation along $\boldsymbol{c}$-axis.

The pyramidal slip could not be guaranteed by size-gradient itself, since the most grains are still larger than the critical size to trigger isotropic deformation [13]. However, the orientation gradient could generate the internal stresses across layers along thickness, which can further reduce the stress anisotropy to trigger pyramidal slip. Under the collective effects, pyramidal slips can be smoothly activated and thus both dislocation density and interaction are enhanced. The pyramidal slip has been predicted by stress relaxation investigation (Fig. 6), further directly observed by TEM (Fig. 12). The analysis based on the pole figures has shown that the pyramidal slip might even occurs at the entirety of thickness, since the contraction feature remains to the center area (Fig. 11).

\section{Conclusions}

A dual gradient microstructure was obtained in AZ31 magnesium alloy through SMAT processing, where the microstructural size and crystallographic orientation change gradually along thickness. The deformation mechanisms and corresponding influence to mechanical properties are investigated. The main conclusions are drawn as follows:

(1) The size and orientation gradients were generated together in magnesium alloy with thickness of $1.7 \mathrm{~mm}$. Attributed from the nucleation and interaction of $\{10 \overline{1} 2\}$ extensive twins and dislocations, the average microstructural size decreases from $2.5 \mu \mathrm{m}$ at center area to about $200 \mathrm{~nm}$ near treated surface, while the $c$-axes gradually tilt from being parallel with normal direction towards inclined with it.

(2) The yield stress of dual gradient microstructure is increased by 4 times as compared with its as-received sample, and 6.5\% uniform elongation is retained at the same time. Meanwhile, the diffusive necking appears in dual gradient sample, leading to $5 \%$ postuniform elongation, which is always negligible in magnesium alloys. Furthermore, the ductility of dual gradient microstructure is higher than that of its constituents, i.e., the gradient layer and corresponding core.
(3) The size refinement through the entirety of thickness significantly reduces the yield stress anisotropy for pyramidal slip, and hence improves its propensity to be activated during tensile deformation. The pyramidal slip will then provide a component vector along $\boldsymbol{c}$ axis, accommodating the incompatibility from basal and prismatic $\langle a\rangle$ slips, and thus make the tensile deformation more uniformly.

(4) The dominant deformation modes change gradually from basal to prismatic slip from treated surface to center area due to orientation gradient. This orientation gradient will raise internal stresses across layers along thickness, which could further lower down the yield stress anisotropy for pyramidal slip besides the contribution of size refinement. The pyramidal slip has been activated along the entirety of thickness. Together with strain gradient and back stress effects, the dual gradient microstructure shows a well combination between strength and ductility.

\section{Acknowledgements}

The authors would like to appreciate in advance for the financial support by the National Natural Science Foundation of China (NSFC) (Grant nos. 51301187, 11572328, 11222224 and 11472286), the Strategic Priority Research Program of the Chinese Academy of Sciences (Grant no. XDB22040500) and the 973 Programs (Grant nos. 2012CB932203, 2012CB937500 and 6138504).

\section{References}

[1] J.C. Williams, E.A. Starke Jr, Acta Mater. 51 (2003) 5775-5799.

[2] C.C. Koch, Nanostructured Materials: Processing, Properties and Applications, William Andrew, 2006.

[3] M. Nastasi, D.M. Parkin, H. Gleiter, Mechanical Properties and Deformation Behavior of Materials having Ultra-fine Microstructures, Springer Science \& Business Media, 2012.

[4] J. Koike, T. Kobayashi, T. Mukai, H. Watanabe, M. Suzuki, K. Maruyama, K. Higashi, Acta Mater. 51 (2003) 2055-2065.

[5] S.R. Agnew, Ö. Duygulu, Int. J. Plast. 21 (2005) 1161-1193.

[6] K. Máthis, K. Nyilas, A. Axt, I. Dragomir-Cernatescu, T. Ungár, P. Lukáč, Acta Mater. 52 (2004) 2889-2894.

[7] T. Al-Samman, X. Li, S.G. Chowdhury, Mater. Sci. Eng. A 527 (2010) 3450-3463.

[8] B. Li, E. Ma, Phys. Rev. Lett. 103 (2009) 035503.

[9] G. Proust, C.N. Tomé, A. Jain, S.R. Agnew, Int. J. Plast. 25 (2009) 861-880.

[10] M. Barnett, Mater. Sci. Eng. A 464 (2007) 8-16.

[11] S. Ando, H. Tonda, Mater. Trans. JIM 41 (2000) 1188-1191.

[12] T. Al-Samman, Acta Mater. 57 (2009) 2229-2242.

[13] Q. Yu, L. Qi, R.K. Mishra, J. Li, A.M. Minor, Proc. Natl. Acad. Sci. USA 110 (2013) 13289-13293.

[14] J.A. Sharon, Y. Zhang, F. Mompiou, M. Legros, K.J. Hemker, Scr. Mater. 75 (2014) $10-13$.

[15] F. Kang, J.Q. Liu, J.T. Wang, X. Zhao, Scr. Mater. 61 (2009) 844-847.

[16] Y. Wei, Y. Li, L. Zhu, Y. Liu, X. Lei, G. Wang, Y. Wu, Z. Mi, J. Liu, H. Wang, Nat. Commun. 5 (2014).

[17] T. Fang, W. Li, N. Tao, K. Lu, Science 331 (2011) 1587-1590.

[18] X. Wu, P. Jiang, L. Chen, F. Yuan, Y.T. Zhu, Proc. Natl. Acad. Sci. USA 111 (2014) 7197-7201.

[19] X. Wu, P. Jiang, L. Chen, J. Zhang, F. Yuan, Y. Zhu, Mater. Res. Lett. 2 (2014) $185-191$.

[20] J. Moering, X. Ma, J. Malkin, M. Yang, Y. Zhu, S. Mathaudhu, Scr. Mater. 122 (2016) 106-109.

[21] X. Wu, M. Yang, F. Yuan, G. Wu, Y. Wei, X. Huang, Y. Zhu, Proc. Natl. Acad. Sci. USA 112 (2015) 14501-14505.

[22] F. Bachmann, R. Hielscher, H. Schaeben, Solid State Phenom., Trans. Tech. Publ. (2010) 63-68.

[23] M.R. Barnett, S. Jacob, B.F. Gerard, J.G. Mullins, Scr. Mater. 59 (2008) 1035-1038.

[24] X.L. Wu, M.X. Yang, F.P. Yuan, L. Chen, Y.T. Zhu, Acta Mater. 112 (2016) 337-346.

[25] E. Ma, Y. Wang, Q. Lu, M. Sui, L. Lu, K. Lu, Appl. Phys. Lett. 85 (2004) 4932-4934.

[26] U. Kocks, H. Mecking, Prog. Mater. Sci. 48 (2003) 171-273.

[27] Y.M. Wang, A.V. Hamza, E. Ma, Appl. Phys. Lett. 86 (2005) 241917.

[28] L. Chen, F. Yuan, P. Jiang, J. Xie, X. Wu, Mater. Sci. Eng. A 618 (2014) 563-571.

[29] L. Chen, F. Yuan, P. Jiang, X. Wu, Mater. Sci. Eng. A 551 (2012) 154-159.

[30] D. Caillard, J.-L. Martin, Thermally Activated Mechanisms in Crystal Plasticity, Elsevier, 2003.

[31] Z. Wu, W. Curtin, Nature 526 (2015) 62-67.

[32] H. Mecking, U.F. Kocks, Acta Mater. 29 (1981) 1865-1875.

[33] K. Lu, J. Lu, Mater. Sci. Eng. A 375 (2004) 38-45.

[34] R. Sánchez-Martín, M. Pérez-Prado, J. Segurado, J. Molina-Aldareguia, Acta Mater. 93 (2015) 114-128.

[35] W.B. Hutchinson, M.R. Barnett, Scr. Mater. 63 (2010) 737-740. 
[36] M.S. Tsai, C.P. Chang, Mater. Sci. Technol. 29 (2013) 759-763.

[37] E. Lilleodden, Scr. Mater. 62 (2010) 532-535.

[38] J. Ye, R.K. Mishra, A.K. Sachdev, A.M. Minor, Scr. Mater. 64 (2011) 292-295.

[39] A. Jain, O. Duygulu, D.W. Brown, C.N. Tomé, S.R. Agnew, Mater. Sci. Eng. A 486 (2008) 545-555.

[40] J.A. del Valle, F. Carreño, O.A. Ruano, Acta Mater. 54 (2006) 4247-4259.

[41] W. Yuan, S.K. Panigrahi, J.Q. Su, R.S. Mishra, Scr. Mater. 65 (2011) 994-997.
[42] G. Bhargava, W. Yuan, S. Webb, R.S. Mishra, Metall. Mater. Trans. A 41 (2010) 13-17.

[43] W. Yuan, R.S. Mishra, B. Carlson, R.K. Mishra, R. Verma, R. Kubic, Scr. Mater. 64 (2011) 580-583

[44] S.M. Razavi, D.C. Foley, I. Karaman, K.T. Hartwig, O. Duygulu, L.J. Kecskes, S.N. Mathaudhu, V.H. Hammond, Scr. Mater. 67 (2012) 439-442.

[45] M. Yang, Y. Pan, F. Yuan, Y. Zhu, X. Wu, Mater. Res. Lett. (2016) 1-7. 